High Energy Phenomena in Relativistic Outflows III (HEPRO III)

International Journal of Modern Physics: Conference Series

Vol. 8 (2012) 360-363

(C) World Scientific Publishing Company

DOI: $10.1142 / \mathrm{S} 2010194512004898$

\title{
EFFICIENCY OF INTERNAL SHOCKS IN MAGNETIZED RELATIVISTIC JETS
}

\author{
PETAR MIMICA \\ Departament d'Astronomia $i$ Astrofísica, Universitat de Valencia \\ C/ Dr. Moliner, 50, E-46100 Burjassot (Valencia), Spain \\ Petar.Mimica@uv.es \\ MIGUEL ANGEL ALOY \\ Departament d'Astronomia $i$ Astrofísica, Universitat de Valencia \\ C/ Dr. Moliner, 50, E-46100 Burjassot (Valencia), Spain \\ Miguel.A.Aloy@uv.es
}

\begin{abstract}
We study the dynamic and radiative efficiency of conversion of kinetic-tothermal/magnetic energy by internal shocks in relativistic magnetized outflows. A parameter study of a large number of collisions of cylindrical shells is performed. We explore how, while keeping the total flow luminosity constant, the variable fluid magnetization influences the efficiency and find that the interaction of shells in a mildly magnetized jet yields higher dynamic, but lower radiative efficiency than in a non-magnetized flow. A multi-wavelength radiative signature of different shell magnetizations is computed assuming that relativistic particles are accelerated at internal shocks.
\end{abstract}

Keywords: Magnetohydrodynamics (MHD); Shock waves; radiation processes: nonthermal; gamma-ray bursts; relativistic jets: blazars

\section{Introduction}

The radiation observed in the relativistic outflows of blazars and gamma-ray bursts (GRBs) shows a high degree of variability. Although the radiation energy and time scales are different for both classes of objects, the underlying physics is probably very similar. The internal shock scenario ${ }^{1}$ has been used to explain the variability of blazars ${ }^{2,3}$ and GRBs: ${ }^{4,5,6}$ inhomogeneities in the outflow cause different parts (shells) to collide and produce the internal shock waves. The dissipation originated at such shocks has been studied by means of relativistic (magneto)hydrodynamic simulations ${ }^{3,7,8,9}$ in non-/weakly magnetized outflows, and has shown the complexity of shell interactions, not taken fully into account by simpler analytic models. ${ }^{2,4,5,6}$

These studies did not address the highly magnetized regime of shell interactions, particularly, they did not consider whether it is only kinetic or also the magnetic energy which can be used to power the emission. In a previous work ${ }^{10}$ we study the efficiency of conversion of the kinetic to thermal and magnetic energy (we call it dynamic efficiency). The main results of that study show that 1) the dynamic 
efficiency is highest if the shells are moderately magnetized (magnetization $\sigma \approx 0.1$ ), 2 ) if the relative difference in magnetization between colliding shells is $\approx 5-100$, the efficiency of a single collision can be as high as $20 \%$ and 3 ) multiple shell collisions can raise the efficiency to even higher values (close to 100\%).

In this work we discuss some preliminary results of the upcoming paper ${ }^{11}$ on the radiative efficiency of the shell collisions. More precisely, we discuss the definitions of the efficiency and the radiative signature of strongly magnetized shell collisions.

\section{Dynamic and radiative efficiency}

In our previous work ${ }^{10}$, we define the dynamic thermal and magnetic efficiency $\left(\varepsilon_{T}\right.$ and $\varepsilon_{M}$, respectively) as a fraction of the total initial energy in the shells which is converted into an thermal and magnetic energy in the merged shell, respectively. We also defined the total dynamic efficiency as $\varepsilon:=\varepsilon_{T}+\varepsilon_{M}$.

The radiative efficiency $\varepsilon_{R}$ can be defined as a fraction of the total initial energy in the shells converted into radiation. Assuming that only a fraction $\varepsilon_{e}$ of the thermal energy dissipated at the shock can be transferred to non-thermal particles and later radiated, we can write

$$
\varepsilon_{R}=f_{R} \varepsilon_{e} \varepsilon
$$

where $f_{R}:=\varepsilon_{T} /\left(\varepsilon_{T}+\varepsilon_{M}\right)$. The reason that we only take the thermal energy as being available for radiation is that we do not consider any magnetic dissipation processes (ideal limit). Figure 1 shows the contours of $f_{R}$ and the filled contours of $\varepsilon$ as a function of the shell magnetization. We denote the faster and slower shells by the subscripts $L$ and $R$, respectively. The shell Lorentz factors are $\Gamma_{L}=20$ and $\Gamma_{R}=10$, which corresponds to a typical blazar jet. It can be seen that, although the dynamic efficiency has its maximum in the region $\left(\sigma_{L} \simeq 1, \sigma_{R} \simeq 0.1\right)$, there, the radiative efficiency is lower than for the weakly magnetized shells $\left(f_{R} \leq 0.3\right.$ there, as opposed to $f_{R} \geq 0.9$ when $\left.\sigma_{L, R} \leq 0.01\right)$. The plot has been obtained using a grid of $10^{6}$ different values of $\left(\sigma_{L}, \sigma_{R}\right)$ pairs and then solving Riemann problems using method described in our previous work ${ }^{10}$.

\section{Radiative signature}

Figure 2 shows the light curves for two models, $\left(\sigma_{L}=10^{-6}, \sigma_{R}=10^{-6}\right.$ ) (full lines) and $\left(\sigma_{L}=1, \sigma_{R}=0.1\right)$ (dashed lines). Shown are the optical (thick lines) and X-ray (thin lines) light curves. The method for the computation of emission will be described in our upcoming article ${ }^{11}$, and is very similar to the ones already used to study multiwavelength light curves from non-magnetized shell collisions ${ }^{12,13}$. We take into account the synchrotron and the synchrotron self-Compton (SSC) processes. The optical radiation is dominated by the synchrotron process, while the $\mathrm{X}$-ray radiation is dominated by the SSC. Once the shocks cross the shells the optical emission peaks and drops afterward, while the SSC emission peaks at later times 


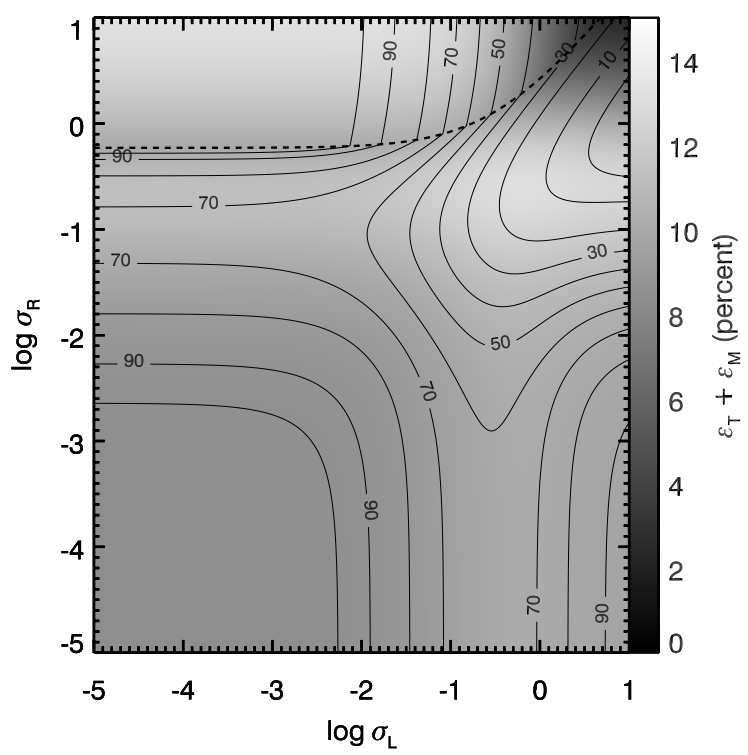

Fig. 1. Contours of $f_{R}$ (solid lines) plotted over the dynamic efficiency distribution (grey shades) as a function of left (faster) and right (slower) shell magnetizations. The contours show $f_{R}$ in per cent with the values $1,5,10,20,30,40,50,60,70,80,90,95$ and 100 . A value $\varepsilon_{e}=1$ is considered.

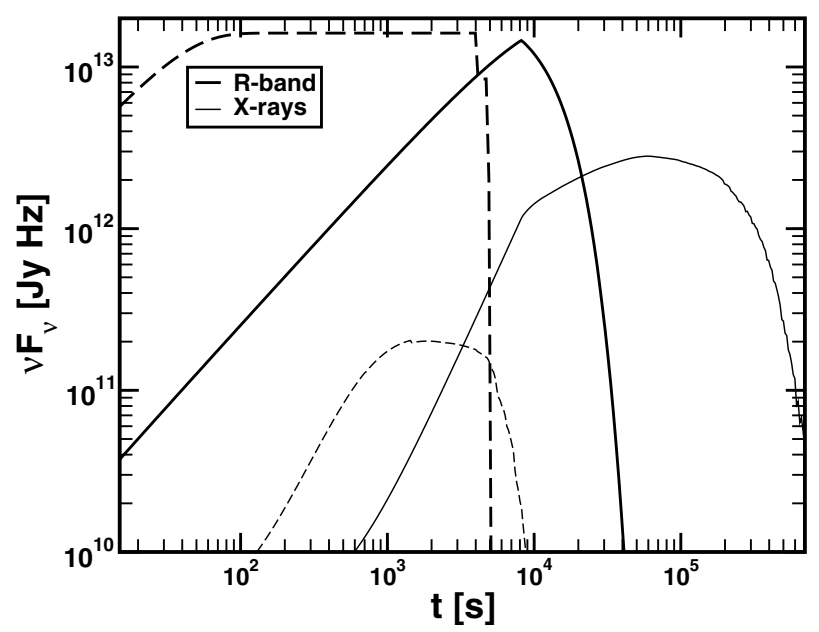

Fig. 2. Optical (thick lines) and X-ray (thin lines) light curves for the models $\left(\sigma_{L}=10^{-6}, \sigma_{R}=\right.$ $\left.10^{-6}\right)$ (full lines) and $\left(\sigma_{L}=1, \sigma_{R}=0.1\right)$ (dashed lines).

because of the delay caused by the travel time of the seed synchrotron photons, which are scattered to X-ray frequencies.

The difference between the non-magnetized (full lines) and the magnetized model (dashed lines) is both in the intensity of the emitted radiation, as well as in the shape of the light curve. The magnetized model optical light curve is almost constant until 
the sharp drop-off, corresponding to the point when the shocks cross the shells. Due to the high magnetic field the electrons are fast cooling and the radiation drops very soon after the shocks stop accelerating fresh particles. In the non-magnetized case the magnetic field is low enough that the electrons are slow cooling and the light curve peaks when the shocks cross the shell, and then decays gently as the electrons cool. The SSC emission from the magnetized model is much lower than the one from the non-magnetized one due to the lower density of the scattering electrons (both models have the same total energy, but in the magnetized case a substantial part of the energy is carried by the magnetic field, while in the non-magnetized case all the energy is in the baryons).

\section{Conclusions}

We studied the radiative efficiency and the radiative signature of the collisions of arbitrarily magnetized shells in the variable outflow of blazars and GRBs. Our results are preliminary, but they show that, while the dynamic efficiency in magnetized outflows can be higher, the radiative efficiency (at least in the internal shocks model) is probably much lower. The multi-wavelength light curve also shows substantial differences between the two cases, notably in the shape of the optical light curve.

\section{Acknowledgments}

PM and MA acknowledge the support from the European Research Council (grant CAMAP-259276), and the partial support of grants AYA2007-67626-C0301, CSD2007-00050, and PROMETEO-2009-103. The authors thankfully acknowledge the computer resources, technical expertise and assistance provided by the Barcelona Supercomputing Center - Centro Nacional de Supercomputación.

\section{References}

1. M J. Rees and P. Meszaros, Astrophys. J. Lett. 430, L93 (1994).

2. M. Spada et al., Mon. Not. R. Astron. Soc. 325, 1559 (2001).

3. P. Mimica et al., Astron. Astrophys. 418, 947 (2004).

4. S. Kobayashi, T. Piran and R. Sari, Astrophys. J. 490, 92 (1997).

5. F. Daigne and R. Mochkovitch, Mon. Not. R. Astron. Soc. 296, 275 (1998).

6. Ž. Bošnjak, F. Daigne and G. Dubus, Astron. Astrophys. 498, 677 (2009).

7. M. Kino, A. Mizuta and S. Yamada, APJ 611, 1021 (2004)

8. P. Mimica et al., Astron. Astrophys. 441, 103 (2005).

9. P. Mimica, M. A. Aloy and E. Müller, Astron. Astrophys. 46693 (2007).

10. P. Mimica and M. A. Aloy, Mon. Not. R. Astron. Soc. 401, 525 (2010).

11. P. Mimica and M. A. Aloy, in preparation (2011).

12. M. Böttcher and C. Dermer, Astrophys. J. 711, 445 (2010).

13. M. Joshi M. and M. Böttche, Astrophys. J. 727, 21 (2011). 